\title{
Priorización semi-cuantitativa basada en evidencia de enfermedades bacterianas zoonóticas presentes en la interfaz cerdos silvestres (Sus scrofa), cerdos domésticos y humanos en Colombia
}

\author{
Semi-quantitative prioritization based on evidence of zoonotic bacterial \\ diseases present at the interface between wild pigs (Sus scrofa), \\ domestic pigs and humans in Colombia
}

\author{
Natalia Martínez-Granados ${ }^{1}$, Jessica Mateus-Anzola ${ }^{1,5}$, Diana Benavides-Arias ${ }^{2,6}$, \\ Diego Soler-Tovar ${ }^{3}$, Patricia Hernández-Rodríguez ${ }^{4}$
}

\section{Resumen}

\begin{abstract}
El objetivo del estudio fue priorizar las enfermedades bacterianas zoonóticas presentes en la interfaz cerdos silvestres (Sus scrofa), cerdos domésticos y humanos en Colombia. Se realizó una revisión de sistemas de indexación y resúmenes (SIR) y de datos del Sistema Mundial de Información Zoosanitaria (WAHID/WAHIS). Se empleó el método de priorización semi-cuantitativo basado en la evidencia con 27 criterios en cinco categorías: epidemiología, prevención/control, economía/comercio, salud pública y so-
\end{abstract}

\footnotetext{
${ }^{1}$ Semillero de Investigación Una Salud, Programa de Medicina Veterinaria, Facultad de Ciencias Agropecuarias, Universidad de La Salle, Bogotá DC, Colombia

${ }^{2}$ Grupo de Investigación Epidemiologia y Evaluación en Salud Pública, Facultad de Medicina, Departamento de Salud Pública, Universidad Nacional de Colombia, Bogotá, Colombia

${ }^{3}$ Grupo de Epidemiologia y Salud Pública, Facultad de Ciencias Agropecuarias, Universidad de La Salle, Bogotá DC, Colombia

${ }^{4}$ Grupo de Investigación BIOMIGEN, Escuela de Ciencias Básicas y Aplicadas, Universidad de La Salle, Bogotá DC, Colombia

${ }_{5}^{5}$ Laboratorio de Ecología de Enfermedades y Una Salud, Facultad de Medicina Veterinaria y Zootecnia, Universidad Nacional Autónoma de México, Ciudad de México, México

${ }^{6}$ E-mail: dbenavidesa@unal.edu.co
}

Estudio financiado con recursos del Semillero de Investigación Una Salud y el Grupo de Epidemiologia y Salud Pública de la Universidad de La Salle (Bogotá, Colombia)

Recibido: 17 de diciembre de 2020

Aceptado para publicación: 2 de octubre de 2021

Publicado: 22 de diciembre de 2021

CLos autores. Este artículo es publicado por la Rev Inv Vet Perú de la Facultad de Medicina Veterinaria, Universidad Nacional Mayor de San Marcos. Este es un artículo de acceso abierto, distribuido bajo los términos de la licencia Creative Commons Atribución 4.0 Internacional (CC BY 4.0) [https:// creativecommons.org/licenses/by/4.0/deed.es] que permite el uso, distribución y reproducción en cualquier medio, siempre que la obra original sea debidamente citada de su fuente original 
ciedad, con valores entre 0 (sin riesgo) y 7 (riesgo severo). La salmonelosis (148 puntos) y la tuberculosis (148) presentaron potencial zoonótico alto, con evidencia de mayor puntuación en los criterios de epidemiología, salud pública y sociedad; seguido de yersiniosis (140) y brucelosis (127) con potencial zoonótico medio, y finalmente leptospirosis (119) y pasteurelosis (114) con potencial zoonótico bajo.

Palabras clave: enfermedades transmisibles, Salmonella, Mycobacterium tuberculosis, salud pública, vigilancia epidemiológica, Sus scrofa

\section{Abstract}

The aim of this study was to prioritize the zoonotic bacterial diseases present in the interface between wild pigs (Sus scrofa), domestic pigs and humans in Colombia. A review of indexing and abstracting systems and data from the World Animal Health Information System (WAHID/WAHIS) was carried out. The semi-quantitative method of prioritization based on evidence was used with 27 criteria in five categories: epidemiology, prevention/control, economy/trade, public health and society, with values between 0 (no risk) and 7 (severe risk). Salmonellosis (148 points) and tuberculosis (148) presented high zoonotic potential, with evidence of a higher score in epidemiology, public health and society criteria; followed by yersiniosis (140) and brucellosis (127) with medium zoonotic potential, and finally leptospirosis (119) and pasteurellosis (114) with low zoonotic potential.

Key words: communicable diseases, Salmonella, Mycobacterium tuberculosis, public health, epidemiological surveillance, Sus scrofa

\section{INTRODUCCIÓN}

Las enfermedades zoonóticas transmitidas por animales domésticos han sido ampliamente estudiadas; sin embargo, el conocimiento sobre la interfaz animales silvestresanimales domésticos y humanos es aún limitado (Miller et al., 2013). Igualmente, la falta de claridad en el proceso ecológico de transmisión de enfermedades entre animales de vida silvestre y el ganado dificulta el control de las enfermedades infecciosas en estas poblaciones (Barasona et al., 2014).

En Estados Unidos, de 86 enfermedades reportadas en aves, rumiantes, porcinos y lagomorfos, el 79\% tiene un componente de vida silvestre asociado con la transmisión, el mantenimiento o el ciclo de vida del pató- geno y, de esas enfermedades, $40 \%$ tienen potencial zoonótico (Miller et al., 2013). Además, se estima que $75 \%$ de las enfermedades infecciosas en humanos son de origen zoonótico, en tanto que $74.5 \%$ de las patologías reemergentes se asocian con zoonosis. Así mismo, Otte y Pica-Ciamarra (2021) reportaron que el $72 \%$ de lasenfermedades zoonóticas se originan en la vida silvestre. Por ello, la vigilancia sanitaria de la fauna silvestre debe considerarse tan relevante como la de los animales domésticos con el fin de proteger tanto la salud de los animales como del humano (WAHIS, s.f.).

Los cerdos silvestres (Sus scrofa) son una especie de relevancia epidemiológica, debido a que pueden servir como reservorios de múltiples enfermedades transmisibles, ta- 
les como tuberculosis, leptospirosis y brucelosis (Rovid et al., 2010), las cuales impactan significativamente en la industria porcícola causando grandes pérdidas económicas, debido principalmente a la alta mortalidad, al sacrificio como medida de control de enfermedades infecciosas y a las restricciones en el comercio nacional e internacional de cerdos y sus productos cárnicos (OIE, 2016).

E1 incremento de la densidad poblacional de cerdos silvestres en el ámbito mundial es indicativo no solo de un mayor número de huéspedes disponibles para la transmisión de enfermedades, sino también de un aumento de la tasa de contacto entre ellos (Acevedo et al., 2016), generando problemas en las explotaciones extensivas por aumento de riesgos sanitarios que llevan a pérdidas económicas (Carpinetti et al., 2014). Por lo tanto, priorizar las enfermedades bacterianas zoonóticas relacionadas con la interfaz silvestre es relevante, máxime cuando la población porcina en Colombia cuenta con más de seis millones de animales en cerca de 231 mil predios, de los cuales $94.9 \%$ son predios de traspatio y los restantes corresponden a predios tecnificados (ICA, 2021), principalmente en Antioquia (35.5\%), Cundinamarca (10.6\%) y Valle del Cauca (9.0\%), departamentos en los cuales se concentra cerca del $55.2 \%$ de la población porcina. En estas regiones se reporta salmonelosis, brucelosis y leptospirosis como las enfermedades infecciosas más comunes en cerdos (FAO, 2010).

El objetivo del presente estudio fue priorizar las enfermedades zoonóticas bacterianas en la interfaz cerdos silvestres (Sus scrofa), cerdos domésticos y humanos en Colombia mediante la revisión de sistemas de indexación y resúmenes (SIR), datos del Sistema Mundial de Información Zoosanitaria (WAHIS, s.f.) y el método de priorización semi-cuantitativo basado en la evidencia.

\section{Materiales y MéTodos}

Se realizó la búsqueda de información mediante la revisión de sistemas de indexación y resúmenes (SIR): PubMed, Sage Journals, ScienceDirect y Scopus, así como la información reportada por los sistemas de vigilancia de orden nacional e internacional, teniendo criterios de inclusión como: el artículo debe responder a una o más preguntas de cada criterio, la referencia debe haber sido publicada en revistas científicas indexadas, debe existir reportes de la enfermedad en cerdos silvestres-domésticos-humanos y se debe presentar en Colombia. Para esto, se utilizaron palabras clave como Salmonella, Brucella, Mycobacterium, Pasteurella, Leptospira, Yersina enterocolitica, salmonelosis, brucelosis, tuberculosis, pasteurelosis, leptospirosis y yersiniosis en cerdos silvestres-cerdos domésticos entre los años 1984 y 2015, tanto en español como en inglés. Además, se utilizaron los datos del Sistema Mundial de Información Zoosanitaria (WAHIS, s.f.). La escala de ponderación de cada criterio se le adjudicó un coeficiente entre 0 y 7 de acuerdo con su impacto medido con base en la información científica, donde cero es ningún impacto y siete mayor impacto.

Posteriormente, se llevó a cabo el método de priorización semi-cuantitativo basado en evidencia reportado en la literatura (Humblet et al., 2012; Rist et al., 2014; Cito et al., 2015), en el cual se tomaron en cuenta 27 criterios clasificados en cinco categorías (Cuadros 1, 2 y 3). Cada criterio se valoró con 0-7 puntos, correlacionados con su severidad, teniendo una puntuación de 0 los agentes no zoonóticos. Así, la mayor puntuación la tienen los agentes con más impactos, obteniéndose una sumatoria máxima de 189. Finalmente, se realizó la priorización por categoría de los múltiples criterios de enfermedades en Sus scrofa, dividiéndolo en tres grupos: potencial zoonótico alto, medio y bajo, según la sumatoria final de cada enfermedad. 
Cuadro 1. Categorías y descripción de criterios de Humblet et al. (2012), Rist et al. (2014) y Cito et al. (2015), según el método de priorización semi-cuantitativa (Parte I)

\begin{tabular}{|c|c|c|}
\hline Categoría & Criterio & $\begin{array}{l}\text { Descripción } \\
\text { (puntuación: 0-7) }\end{array}$ \\
\hline \multicolumn{3}{|c|}{ Epidemiología } \\
\hline & \multirow[t]{4}{*}{ Tasa de morbilidad } & No morbilidad (0) \\
\hline & & Tasa de morbilidad $<1 \%$ (1) \\
\hline & & Tasa de morbilidad < 50\% (4) \\
\hline & & Tasa de morbilidad > 90\% (7) \\
\hline & \multirow[t]{4}{*}{ Tasa de mortalidad } & No mortalidad: $(0)$ \\
\hline & & Tasa de mortalidad $<1 \%$ (1) \\
\hline & & Tasa de mortalidad $<50 \%$ (4) \\
\hline & & Tasa de mortalidad $>90 \%(7)$ \\
\hline & \multirow[t]{5}{*}{ Especificidad del patógeno } & No se encuentra reportes $(0)$ \\
\hline & & Sólo se presenta en el cerdo (1) \\
\hline & & Involucra otros animales domésticos (4) \\
\hline & & Multi-húesped (7) \\
\hline & & Involucra animales silvestres (7) \\
\hline & \multirow[t]{4}{*}{ Modo de transmisión } & Indirecta (5) \\
\hline & & $\begin{array}{l}\text { Directa (no necesita ni huésped ni vector ni } \\
\text { reservorio): }\end{array}$ \\
\hline & & Vertical (6) \\
\hline & & Horizontal (7) \\
\hline & \multirow{3}{*}{$\begin{array}{l}\text { Persistencia en el medio } \\
\text { ambiente }\end{array}$} & Se inactiva rápidamente en el ambiente (1) \\
\hline & & Sobreviven horas/días en el ambiente (3-4) \\
\hline & & $\begin{array}{l}\text { Sobreviven durante mucho tiempo en el medio } \\
\text { ambiente (7) }\end{array}$ \\
\hline & \multirow{3}{*}{$\begin{array}{l}\text { Enfermedad clínica en } \\
\text { cerdos }\end{array}$} & No se presentan signos clínicos o son portadores (1) \\
\hline & & Enfermedad subclínica (4) \\
\hline & & Presentación de signos clínicos (7) \\
\hline & \multirow{3}{*}{$\begin{array}{l}\text { Enfermedad clínica en } \\
\text { animales silvestres }\end{array}$} & No se presentan signos clínicos o portadores (1) \\
\hline & & Enfermedad subclínica (4) \\
\hline & & Presentación de signos clínicos (7) \\
\hline & \multirow{2}{*}{$\begin{array}{l}\text { Evidencia circulación en } \\
\text { Colombia }\end{array}$} & Sin evidencia de circulación (1) \\
\hline & & Datos epidemiológicos en Colombia (7) \\
\hline \multicolumn{3}{|c|}{ Prevención/control } \\
\hline & \multirow[t]{3}{*}{ Control de reservorio/vector } & Modo de transmisión directa (no hay vector) (0) \\
\hline & & Se realiza control efectivo (1) \\
\hline & & $\begin{array}{l}\text { No se realiza control efectivo o es de difícil control } \\
\text { (7) }\end{array}$ \\
\hline & \multirow[t]{2}{*}{ Vacunación } & Se cuenta con vacuna (1) \\
\hline & & No se cuenta con vacuna (7) \\
\hline & \multirow[t]{2}{*}{ Tratamiento } & Existe tratamiento para la enfermedad (1) \\
\hline & & No existe tratamiento (7) \\
\hline
\end{tabular}


Cuadro 2. Categorías y descripción de criterios de Humblet et al. (2012), Rist et al. (2014) y Cito et al. (2015), según el método de priorización semi-cuantitativa (Parte II)

\begin{tabular}{|c|c|c|}
\hline Categoría & Criterio & $\begin{array}{l}\text { Descripción } \\
\text { (puntuación: 0-7) }\end{array}$ \\
\hline \multicolumn{3}{|c|}{ Economía y comercio } \\
\hline & $\begin{array}{l}\text { Disminución de la } \\
\text { productividad }\end{array}$ & $\begin{array}{l}\text { No hay impacto en la productividad (1) } \\
\text { Hay impacto en la productividad (7) }\end{array}$ \\
\hline & $\begin{array}{l}\text { Limitaciones de } \\
\text { importación-exportación }\end{array}$ & $\begin{array}{l}\text { No hay limitación en el comercio (1) } \\
\text { El impacto no es tan marcado (4) } \\
\text { Hay prohibiciones de exportación e importación (7) }\end{array}$ \\
\hline & $\begin{array}{l}\text { Impacto en sectores } \\
\text { adyacentes (turismo) }\end{array}$ & $\begin{array}{l}\text { No hay impacto en turismo (1) } \\
\text { Hay disminución de turistas nacionales e } \\
\text { internacionales ( } 7 \text { ) }\end{array}$ \\
\hline & $\begin{array}{l}\text { Impacto zoonótico (costo de } \\
\text { la enfermedad) }\end{array}$ & $\begin{array}{l}\text { No existen reportes }(0) \\
\text { No hay evidencia de la enfermedad en humanos (1) } \\
\text { Elevados costos de enfermedades zoonóticas ( } 7)\end{array}$ \\
\hline \multicolumn{3}{|c|}{ Salud pública } \\
\hline & Zoonosis & $\begin{array}{l}\text { No es una enfermedad zoonótica (1) } \\
\text { Es una enfermedad zoonótica ( } 7)\end{array}$ \\
\hline & Clasificación de la zoonosis & $\begin{array}{l}\text { Ciclozoonosis (4) } \\
\text { Zooantroponosis (7) } \\
\text { Antropozoonosis (7) } \\
\text { Metazoonosis (7) }\end{array}$ \\
\hline & $\begin{array}{l}\text { Enfermedad conocida en } \\
\text { humanos }\end{array}$ & $\begin{array}{l}\text { Se desconoce en humanos }(0) \\
\text { Se conoce en humanos }(7)\end{array}$ \\
\hline & Tasa de morbilidad & $\begin{array}{l}\text { No morbilidad }(0) \\
\text { Tasa de morbilidad }<1 \%(1) \\
\text { Tasa de morbilidad }<50 \%(4) \\
\text { Tasa de morbilidad }>90 \%(7)\end{array}$ \\
\hline & Tasa de mortalidad & $\begin{array}{l}\text { No mortalidad }(0) \\
\text { Tasa de mortalidad }<1 \%(1) \\
\text { Tasa de mortalidad }<50 \%(4) \\
\text { Tasa de mortalidad }>90 \%(7)\end{array}$ \\
\hline & Modo de transmisión & $\begin{array}{l}\text { Indirecta (5) } \\
\text { Directa (no necesita ni huésped ni vector ni } \\
\text { reservorio) } \\
\quad \text { Vertical (6) } \\
\quad \text { Horizontal (7) }\end{array}$ \\
\hline & Presencia de plan de control & $\begin{array}{l}\text { Existe plan de control (1) } \\
\text { No existe plan de control (7) }\end{array}$ \\
\hline & Vacunación & $\begin{array}{l}\text { Plan de vacunación (1) } \\
\text { No se cuenta con plan de vacunación (7) }\end{array}$ \\
\hline & Tratamiento & $\begin{array}{l}\text { Se realiza tratamiento médico }(1) \\
\text { No se realiza tratamiento médico }(7)\end{array}$ \\
\hline
\end{tabular}


Cuadro 3. Categorías y descripción de criterios de Humblet et al. (2012), Rist et al. (2014) y Cito et al. (2015), según el método de priorización semi-cuantitativa (Parte III)

\begin{tabular}{lll}
\hline Categoría & Criterio & $\begin{array}{l}\text { Descripción } \\
\text { (puntuación: 0-7) }\end{array}$ \\
\hline Sociedad & Disminución del consumo & $\begin{array}{l}\text { No hay impacto negativo en la industria porcícola } \\
(1)\end{array}$ \\
& $\begin{array}{l}\text { Impacto negativo en el consumo de carne de cerdo } \\
(7)\end{array}$ \\
& Percepción del problema & $\begin{array}{l}\text { Conoce las enfermedades y microorganismos que } \\
\text { afectan al cerdo (1) } \\
\text { por parte del consumidor } \\
\text { Noene conocimiento de las enfermedades que } \\
\text { afectan al cerdo (7) } \\
\text { Impacto en animales } \\
\text { silvestres y la biodiversidad impacto en animales silvestres y }\end{array}$ \\
& $\begin{array}{l}\text { biodiversidad (0) } \\
\text { Impacto negativo en los animales silvestres (7) }\end{array}$ \\
\hline
\end{tabular}

\section{Resultados}

Las dos enfermedades que presentaron un potencial zoonótico alto después de realizar la sumatoria de cada criterio fueron la salmonelosis (148 puntos) y la tuberculosis (148) (Cuadro 4), seguido de yersiniosis (140) y brucelosis (127) con un potencial medio (Cuadro 5) y finalmente, leptospirosis (119) y pasteurelosis (114) con potencial bajo (Cuadros 6). La salmonelosis y tuberculosis, enfermedades priorizadas que presentaron los criterios de puntuación más alta en las categorías de epidemiología, salud pública y sociedad (Cuadro 7), se encuentran directamente relacionadas con una mayor exposición a los factores de riesgo que favorecen la presentación de la enfermedad.

Colombia se encuentra regionalizada según condiciones de producción, mercadeo y topografía (ICA, 2021) en cuatro regiones (Figura 1):

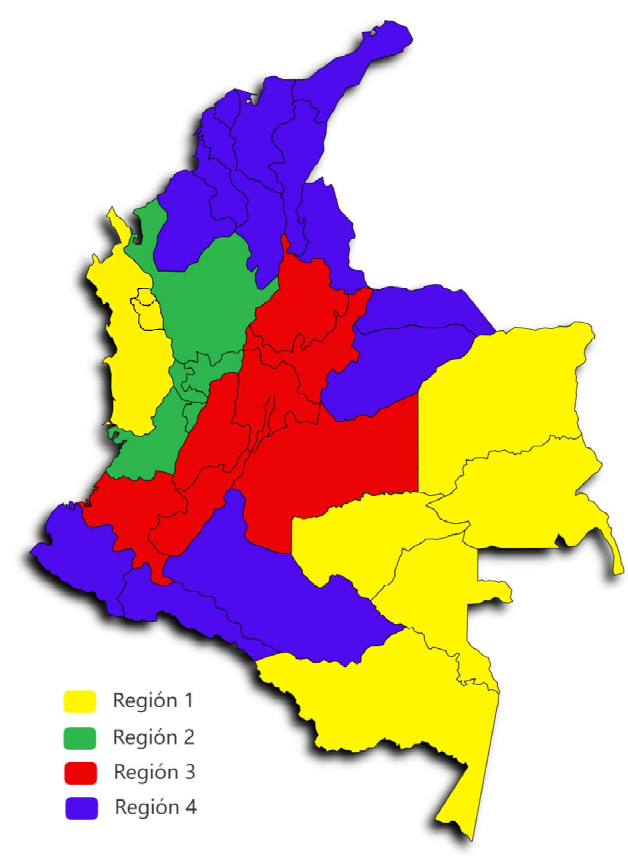

Figura 1. Regiones de Colombia según producción, mercadeo y topografía porcina 
Cuadro 4. Enfermedades bacterianas priorizadas con alto potencial zoonótico

\begin{tabular}{|c|c|c|c|c|}
\hline 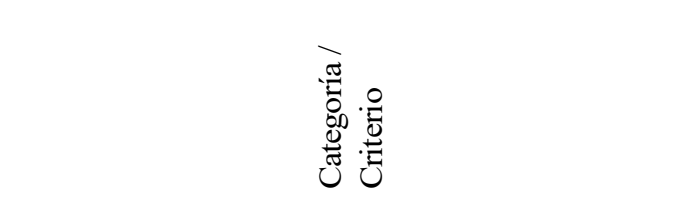 & 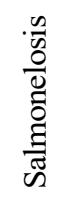 & $\frac{\sqrt{2}}{\sqrt[3]{2}}$ & 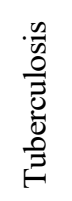 & $\frac{\sqrt{2}}{0}$ \\
\hline \multicolumn{5}{|l|}{ Epidemiologia } \\
\hline Tasa de morbilidad & 7 & Barandiarián et al. & 7 & Clark et al. (2015) \\
\hline Tasa de mortalidad & 7 & $(2015)$ & 4 & \\
\hline Especificidad del patógeno & 7 & $\begin{array}{l}\text { Barasona et al. } \\
\text { (2014) }\end{array}$ & 7 & $\begin{array}{c}\text { De Busser et al. } \\
\text { (2013) }\end{array}$ \\
\hline Modo de transmisión & 5 & $\begin{array}{c}\text { Barandiarián et al. } \\
\text { (2015) }\end{array}$ & 7 & $\begin{array}{c}\text { Dolso y Privado } \\
\text { (2008) }\end{array}$ \\
\hline Persistencia en el medio ambiente & 7 & Barasona et al. & 7 & \\
\hline Enfermedad clínica en cerdos & 7 & $(2014)$ & 7 & \\
\hline Enfermedad clínica en animales silvestres & 7 & $\begin{array}{l}\text { Barasona et al. } \\
\text { (2014); } \\
\text { Barandiarián et al. } \\
\text { (2015) }\end{array}$ & 7 & Elika (2013) \\
\hline Evidencia circulación en Colombia & 7 & Borie et al. (1997) & 7 & Espinal et al. (2006) \\
\hline \multicolumn{5}{|l|}{ Prevención/control } \\
\hline Control de reservorio/vector & 1 & Carreras (2020) & 0 & Elika (2013) \\
\hline Vacunación & 1 & $\begin{array}{c}\text { Carpinetti et al. } \\
\text { (2014) }\end{array}$ & 1 & Ferreira et al. (2012) \\
\hline Tratamiento & 1 & $\begin{array}{c}\text { Carriquiriborde et } \\
\text { al. (2006) }\end{array}$ & 7 & $\begin{array}{c}\text { De Busser et al. } \\
\text { (2013) }\end{array}$ \\
\hline \multicolumn{5}{|l|}{ Economía y comercio } \\
\hline Disminución de la productividad & 7 & $\begin{array}{c}\text { Carpinetti et al. } \\
\text { (2014) }\end{array}$ & 7 & Ferreira et al. (2012) \\
\hline Limitaciones de importación-exportación & 7 & $\begin{array}{c}\text { Noreña y Galvis } \\
(2020)\end{array}$ & 7 & Ferro et al. (2006) \\
\hline Impacto en sectores adyacentes (turismo) & 1 & Céspedes (2005) & 0 & \\
\hline Impacto zoonótico (costo de la enfermedad) & 7 & $\begin{array}{c}\text { Barandiarián et al. } \\
\text { (2015) }\end{array}$ & 7 & $\begin{array}{c}\text { Fredriksson-Ahomaa } \\
\text { et al. (2009) }\end{array}$ \\
\hline \multicolumn{5}{|l|}{ Salud pública } \\
\hline Zoonosis & 7 & Barasona et al. & 7 & Ferreira et al. (2012) \\
\hline Clasificación de la zoonosis & 7 & (2014) & 7 & Ferro et al. (2006) \\
\hline Enfermedad conocida en humanos & 7 & Cárdenas (2000) & 7 & Galindo et al. (2010) \\
\hline Tasa de morbilidad & 7 & Borie et al. (1997) & 1 & Garzón et al. (2008) \\
\hline Tasa de mortalidad & 7 & & 7 & $\begin{array}{c}\text { Fredriksson-Ahomaa } \\
\text { et al. (2009) }\end{array}$ \\
\hline Modo de transmisión & 4 & $\begin{array}{c}\text { Barandiarián et al. } \\
\text { (2015) }\end{array}$ & 7 & Hernández (2015) \\
\hline Presencia de plan de control & 7 & Carreras (2020) & 7 & $\begin{array}{l}\text { Humblet et al. } \\
\qquad \text { (2012) }\end{array}$ \\
\hline Vacunación & 1 & & 1 & Ferreira et al. (2012) \\
\hline Tratamiento & 1 & $\begin{array}{l}\text { Chaparro et al. } \\
\text { (2004) }\end{array}$ & 1 & $(\mathrm{FAO}, 2012 \mathrm{a}, \mathrm{b})$ \\
\hline \multicolumn{5}{|l|}{ Sociedad } \\
\hline Disminución del consumo & 7 & $\begin{array}{l}\text { Chavarrias (2008); } \\
\text { Chávez de la Peña } \\
\text { et al. }(2001)\end{array}$ & 7 & Clark et al. (2015) \\
\hline $\begin{array}{l}\text { Percepción del problema por parte del } \\
\text { consumidor }\end{array}$ & 7 & Cito et al. (2015)) & 7 & \\
\hline $\begin{array}{l}\text { Impacto en animales silvestres y la } \\
\text { biodiversidad }\end{array}$ & 7 & $\begin{array}{l}\text { Barasona et al. } \\
\text { (2014) }\end{array}$ & 7 & Elika (2013) \\
\hline Total & 148 & & 148 & \\
\hline
\end{tabular}


Cuadro 5. Enfermedades bacterianas priorizadas con potencial zoonótico medio

\begin{tabular}{|c|c|c|c|c|}
\hline 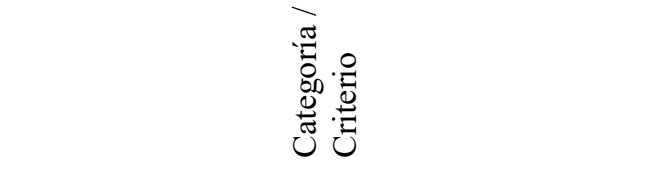 & 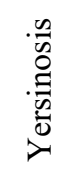 & 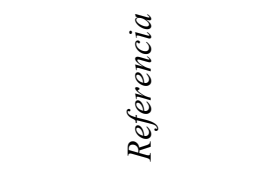 & $\frac{\sqrt[n]{0}}{\frac{0}{0}}$ & 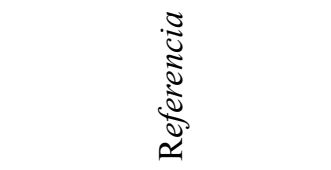 \\
\hline \multicolumn{5}{|l|}{ Epidemiologia } \\
\hline Tasa de morbilidad & 4 & Iglesias (2014) & 4 & Jofré et al. (2006) \\
\hline Tasa de mortalidad & 5 & INS (2016) & 7 & \\
\hline Especificidad del patógeno & 7 & INS (2015) & 7 & Kopper et al. (2009) \\
\hline Modo de transmisión & 7 & Millán (2015) & 7 & Krause (2008) \\
\hline Persistencia en el medio ambiente & 7 & ICA (2011) & 7 & Kumar et al. (2007) \\
\hline Enfermedad clínica en cerdos & 7 & Millán(2015) & 7 & \\
\hline $\begin{array}{l}\text { Enfermedad clínica en animales } \\
\text { silvestres }\end{array}$ & 7 & INS (2015) & 7 & León (1997) \\
\hline Evidencia circulación en Colombia & 7 & $\begin{array}{l}\text { Pulido-Villamarín } \\
\text { et al. (2019) }\end{array}$ & 7 & Leotta et al. (2006) \\
\hline \multicolumn{5}{|l|}{ Prevención/control } \\
\hline Control de reservorio/vector & 0 & Millán (2015) & 0 & Jofré et al. (2006) \\
\hline Vacunación & 7 & INS (2015) & 1 & Rist et al. (2014) \\
\hline Tratamiento & 0 & INS (2016) & 1 & Jofré et al. (2006) \\
\hline \multicolumn{5}{|l|}{ Economía y comercio } \\
\hline Disminución de la productividad & 7 & Iglesias (2014) & 7 & Jofré et al. (2006) \\
\hline Limitaciones de importación-exportación & 7 & $\begin{array}{l}\text { Pulido-Villamarín } \\
\text { et al. (2019) }\end{array}$ & 4 & Luna et al. (1996) \\
\hline Impacto en sectores adyacentes (turismo) & 0 & & 1 & \\
\hline $\begin{array}{l}\text { Impacto zoonótico (costo de la } \\
\text { enfermedad) }\end{array}$ & 7 & INS (2017) & 7 & Kopper et al. (2009) \\
\hline \multicolumn{5}{|l|}{ Salud pública } \\
\hline Zoonosis & 7 & Millán (2015) & 7 & Máttar et al. (2000) \\
\hline Clasificación de la zoonosis & 7 & INS (2017) & 7 & Kumar et al. (2007) \\
\hline Enfermedad conocida en humanos & 5 & Millán (2015) & 7 & León (1997) \\
\hline Tasa de morbilidad & 7 & ICA (2011) & 4 & Jofré et al. (2006) \\
\hline Tasa de mortalidad & 1 & & 4 & Luna et al. (1996) \\
\hline Modo de transmisión & 7 & & 7 & Martín-Hernando et al. \\
\hline Presencia de plan de control & 1 & ICA (2011) & 7 & (2007) \\
\hline Vacunación & 7 & INS (2016) & 1 & Rist et al. (2014) \\
\hline Tratamiento & 0 & & 1 & Martínez et al. (2000) \\
\hline \multicolumn{5}{|l|}{ Sociedad } \\
\hline Disminución del consumo & 7 & Millán (2015) & 1 & Luna et al. (1996) \\
\hline $\begin{array}{l}\text { Percepción del problema por parte del } \\
\text { consumidor }\end{array}$ & 5 & & 7 & \\
\hline $\begin{array}{l}\text { Impacto en animales silvestres y la } \\
\text { biodiversidad }\end{array}$ & 7 & $\begin{array}{l}\text { Jaramillo et al. } \\
\qquad(2014)\end{array}$ & 7 & Máttar et al. (2000) \\
\hline Total & 148 & 140 & & 134 \\
\hline
\end{tabular}


Cuadro 6. Enfermedades bacterianas priorizadas con potencial zoonótico bajo

\begin{tabular}{|c|c|c|c|c|}
\hline 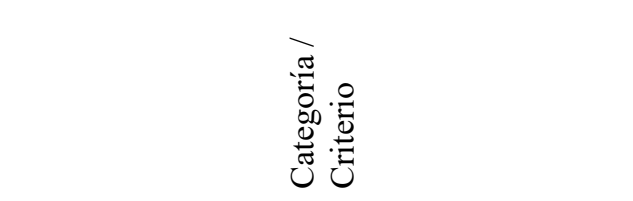 & 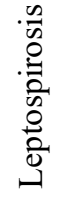 & 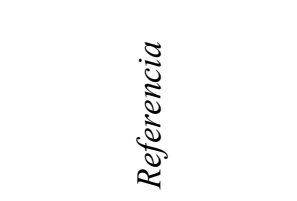 & 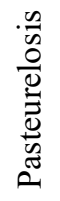 & 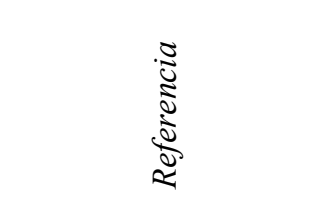 \\
\hline \multicolumn{5}{|l|}{ Epidemiologia } \\
\hline Tasa de morbilidad & 0 & & 6 & Rovid et al. (2010) \\
\hline Tasa de mortalidad & 4 & $\begin{array}{c}\text { De la Sota y } \\
\text { Belgrano }(2004)\end{array}$ & 5 & Sánchez et al. (2015) \\
\hline Especificidad del patógeno & 7 & Miller et al. (2013) & 7 & \\
\hline Modo de transmisión & 7 & DNP (2007) & 7 & Sanchez et al. (2013) \\
\hline Persistencia en el medio ambiente & 7 & $\begin{array}{c}\text { Ministerio de Salud } \\
\text { (2014) }\end{array}$ & 1 & Sánchez (1990) \\
\hline Enfermedad clínica en cerdos & 7 & $\begin{array}{c}\text { Moles-Cervantes et } \\
\text { al. (1998) }\end{array}$ & 7 & SENASA (2009) \\
\hline Enfermedad clínica en animales silvestres & 7 & Nájera et al. (2005) & 7 & Sánchez (1990) \\
\hline Evidencia circulación en Colombia & 7 & $\begin{array}{l}\text { Ng y Sargeant } \\
(2013)\end{array}$ & 7 & $\begin{array}{l}\text { Sepúlveda et al. } \\
\text { (2002) }\end{array}$ \\
\hline \multicolumn{5}{|l|}{ Prevención/control } \\
\hline Control de reservorio/vector & 0 & $\begin{array}{c}\text { De la Sota y } \\
\text { Belgrano }(2004)\end{array}$ & 0 & Sánchez et al. (2013) \\
\hline Vacunación & 1 & Ochoa et al. (2000) & 1 & $\begin{array}{c}\text { Shayegani et al. } \\
\text { (1986) }\end{array}$ \\
\hline Tratamiento & 1 & & 1 & Sánchez et al. (2015) \\
\hline \multicolumn{5}{|l|}{ Economía y comercio } \\
\hline Disminución de la productividad & 7 & OMS (2008) & 7 & Sánchez et al. (2015) \\
\hline Limitaciones de importación-exportación & 7 & FAO (2010) & 1 & Signorini et al. (2006) \\
\hline Impacto en sectores adyacentes (turismo) & 5 & Parra et al. (2003) & 0 & \\
\hline $\begin{array}{l}\text { Impacto zoonótico (costo de la } \\
\text { enfermedad) }\end{array}$ & 7 & Ramírez (2014) & 7 & $\begin{array}{l}\text { Smego y Gallis } \\
\quad(1984)\end{array}$ \\
\hline \multicolumn{5}{|l|}{ Salud pública } \\
\hline Zoonosis & 7 & DNP (2007) & 7 & Sánchez et al. (2013) \\
\hline Clasificación de la zoonosis & 7 & Ramírez (2014) & 7 & $\begin{array}{l}\text { Smego y Gallis } \\
\text { (1984) }\end{array}$ \\
\hline Enfermedad conocida en humanos & 1 & Reyes et al. (2009) & 1 & $\begin{array}{l}\text { Stoffregen et al. } \\
\qquad(2013)\end{array}$ \\
\hline Tasa de morbilidad & 0 & & 0 & \\
\hline Tasa de mortalidad & 5 & Reyes et al. (2009) & 4 & $\begin{array}{l}\text { Center for Food } \\
\text { Security and Public } \\
\text { Health (2009) }\end{array}$ \\
\hline Modo de transmisión & 7 & DNP (2007) & 7 & Sánchez (1990) \\
\hline Presencia de plan de control & 1 & Risco et al. (2013) & 7 & al (2015) \\
\hline Vacunación & 1 & Risco et al. (2013) & 7 & al. $(2015)$ \\
\hline Tratamiento & 1 & FAO (2010) & 1 & $\begin{array}{l}\text { Smego y Gallis } \\
\text { (1984) }\end{array}$ \\
\hline \multicolumn{5}{|l|}{ Sociedad } \\
\hline Disminución del consumo & 7 & FAO (2010) & 1 & Signorini et al. (2006) \\
\hline $\begin{array}{l}\text { Percepción del problema por parte del } \\
\text { consumidor }\end{array}$ & 1 & Rosner et al. (2010) & 1 & \\
\hline $\begin{array}{l}\text { Impacto en animales silvestres y la } \\
\text { biodiversidad }\end{array}$ & 7 & Nájera et al. (2005) & 7 & Sánchez (1990) \\
\hline Total & 119 & & 114 & \\
\hline
\end{tabular}


Cuadro 7. Puntaje de criterios según potencial zoonótico

\begin{tabular}{llc}
\hline $\begin{array}{l}\text { Potencial } \\
\text { zoonótico }\end{array}$ & Enfermedad & $\begin{array}{c}\text { Sumatoria } \\
\text { de criterios }\end{array}$ \\
\hline Alto & Salmonelosis & 148 \\
& Tuberculosis & 148 \\
Medio & Yersiniosis & 140 \\
& Brucelosis & 134 \\
Bajo & Leptospirosis & 119 \\
& Pasteurelosis & 114 \\
\hline
\end{tabular}

- N. 1 (Chocó, Amazonas, Murindó y Vigía del Fuerte en Antioquia, San Andrés y Providencia, Guainía, Guaviare, Vaupés y Vichada). Se caracteriza por una baja población porcina con 33794 animales ( $0.87 \%$ de la población nacional).

- N. 2 (Antioquia, Caldas, Risaralda, Quindío y Valle del Cauca). Cuenta con poblaciones más tecnificadas y núcleos genéticos teniendo una mayor densidad porcina con dos millones de porcinos $(50 \%$ de la población). Presenta implementación de sistemas sanitarios y de bioseguridad. Es una zona de menor riesgo epidemiológico.

- N. 3 (Cundinamarca, Boyacá, Cauca, Meta, Santander, Tolima y Huila). Concentra el $29 \%$ de la población nacional (un millón de animales), presenta sistemas de producción mixtos, con presencia de cerdos silvestres y animales de traspatio, no se manejan esquemas de vacunación estrictos ni se cuentan con tecnologías productivas. Es una zona de alto riesgo epidemiológico.

- N. 4 (Atlántico, Bolívar, Magdalena, Guajira, Cesar, Córdoba, Sucre, Nariño, Norte de Santander, Arauca, Casanare, Caquetá y Putumayo). Presenta alto porcentaje de explotaciones de traspatio (90-95\%) con baja práctica de vacunación, presencia de cerdos silvestres (Figura 2). Tiene una población de 828092 animales $(16.15 \%$ de la población). Es una zona de riesgo epidemiológico.

\section{Discusión}

La priorización de enfermedades como método para la implementación de políticas públicas, se convierte en una herramienta eficaz para enfocar los esfuerzos de los servicios veterinarios y el sector salud en pro de la disminución de enfermedades de origen zoonótico en la interfaz ecosistema-humanoanimal; así como la racionalización de los recursos y su adaptación a contextos epidemiológicos cambiantes utilizada con éxito por varios países (Rist et al., 2014; OIE, 2016); sin embargo, Krause et al. (2008) mencionan que este método puede no funcionar adecuadamente debido a la dificultad en la definición de criterios, la falta de datos por la insuficiente investigación en el campo y la falta de acuerdos con respecto a un sistema de ponderación general. La colaboración intersectorial es una estrategia que puede contribuir a la recopilación de información y monitoreo de tendencias de las enfermedades en poblaciones humanas y animales.

La salmonelosis (148 puntos) y la tuberculosis (148 puntos) fueron las enfermedades con mayor puntuación. En cuanto a la salmonelosis, estudios de prevalencia de anticuerpos de Salmonella spp, entre los años 2001-2004 reportaron 57.8\% de seroprevalencia en granjas porcinas intensivas, lo cual demuestra su importancia en el sector pecuario en términos de costos asumidos por la morbimortalidad, tratamientos y el aumento de la probabilidad de resistencia a los antibióticos; así como para los riesgos en la salud pública. La presencia de este patógeno en poblaciones porcinas domésticas destinadas al consumo humano es la principal causante de la enfermedad transmitida por alimentos y la enfermedad diarreica aguda en Colombia (DNP, 2007). 

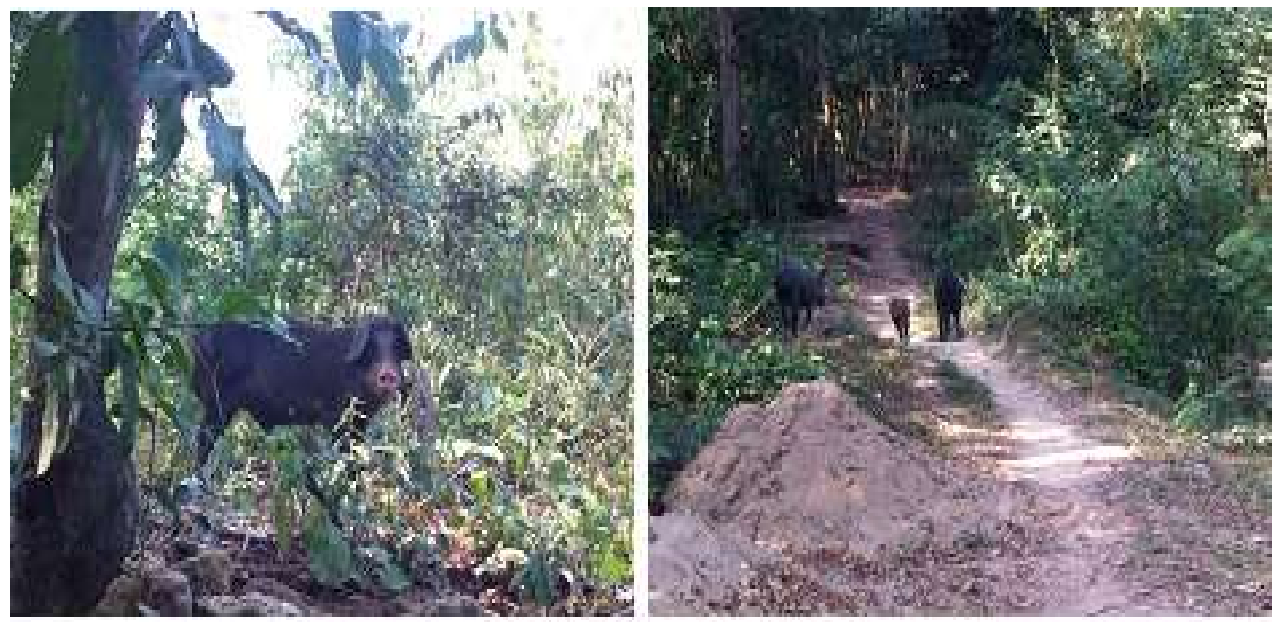

Figura 2. Presencia de cerdos y su interacción en Turbana, Bolívar. Colombia. A. Cerdos silvestres (Sus scrofa); B. Interacción entre cerdo doméstico (centro) y cerdos silvestres (Sus scrofa). Fotos: Diana Benavides Arias

En forma similar, un estudio realizado en 248 predios en 15 departamentos del país evidenció cinco poblaciones porcinas afectadas por Salmonella spp, con una población de riesgo de 318 animales, una incidencia de 9 porcinos $\mathrm{x} 100 \mathrm{y}$ una mortalidad de 55 animales x 1000 (ICA, 2011). La importancia de identificar y realizar la adecuada gestión del riesgo de este patógeno en explotaciones porcícolas radica que en que en muchas ocasiones se encuentran animales asintomáticos, que desarrollan enfermedad subclínica, lo que implica la diseminación silenciosa para trabajadores y consumidores. Por lo cual, determinar la prevalencia real de esta enfermedad a partir de pruebas diagnósticas confiables debe ser una prioridad para los productores y los profesionales involucrados en el diagnóstico humano y animal (Varón, 2020).

Además, en $66.5 \%$ de cerdos silvestres de Italia $(\mathrm{n}=255)$ se encontraron anticuerpos contra Salmonella spp, siendo Salmonella enterica la especie más aislada (24\%) (Zottola, 2013). El Instituto Nacional de Salud de Colombia en 2019 publicó el Informe de Evento de Enfermedades Transmitidas por Alimentos indicando que los principales agentes etiológicos de enfermedades transmitidas por alimentos causantes de enfermedades diarreicas ocasionaron alrededor de 230000 muertes, especialmente por Salmonella enterica no tifoidea, Salmonella Typhi y Taenia solium (INS, 2019a).

En el caso de tuberculosis (148 puntos), los cerdos pueden verse afectados por Mycobacterium tuberculosis, $M$. bovis y $M$. avium (Cano-Terriza et al., 2016). En un estudio en cerdos silvestres (Sus scrofa) de España se encontraron lesiones macroscópicas compatibles con tuberculosis en 83.6\% (105-127) de los animales y lesiones microscópicas en $8.7 \%$ (11 animales), siendo reconocidos por su capacidad de mantener la infección, además de ser un posible reservorio de vida silvestre (Martín-Hernando et al., 2007). En Colombia, hasta la semana epidemiológica 28 de 2016 se notificaron 7287 casos de tuberculosis de todas las formas (INS, 2016) y 12918 casos en 2015 (INS, 
2015), siendo de alta notificación respecto a otras enfermedades.

Por otra parte, aunque la leptospirosis fue de potencial zoonótico bajo (119 puntos), en Colombia es una enfermedad bajo vigilancia epidemiológica, notificándose 2718 casos acumulados para el 2019, concentrándose el 28.7\% de los reportes en Antioquia, 11.6\% en el Valle del Cauca y $11.1 \%$ en Tolima, zonas de gran densidad de población porcícola (INS, 2019b). Esto se convierte en un aspecto de gran importancia para las autoridades sanitarias por el incremento de casos en temporada de lluvias e inundaciones (Pulido-Villa Marín et al., 2019). En países en vías de desarrollo de América Latina y el Caribe (ALC) como Colombia, se presenta un elevado número de casos de enfermedades zoonóticas. Marín (2020) reporta los resultados de una encuesta en ministerios de salud de los 33 países de ALC, donde las zoonosis endémicas prioritarias que se notifican con mayor frecuencia son leptospirosis $(69 \%)$, brucelosis (15\%) y salmonelosis (15\%).

Finalmente, la pasteurellosis (potencial zoonótico bajo) no es una enfermedad de vigilancia epidemiológica a pesar de producir efectos dermatológicos y pulmonares en personas (Sánchez, 1990; Carriquiriborde et al., 2006; Reyes et al., 2009).

Utilizar métodos de priorización de enfermedades zoonóticas resulta de utilidad para contribuir en la identificación de potenciales riesgos para la salud humana y animal. El impacto de estos patógenos en la interfaz de cerdos silvestres, animales domésticos y seres humanos tiene consecuencias directas e indirectas en los costos asociados a la producción pecuaria y ejerce presión sobre los sistemas sanitarios.

\section{Conclusiones}

- La salmonelosis y la tuberculosis fueron las enfermedades con mayor puntuación en la evaluación, según los niveles de severidad planteados en el estudio.

- Se destaca la falencia en la vigilancia epidemiológica en las regiones 3 y 4 de Colombia. Departamentos como Bolívar, Cundinamarca y Tolima podrían desempeñar un rol más activo en la prevención y control de estas enfermedades, dado que son zonas de circulación considerable de la bacterias y de mayor riesgo epidemiológico en poblaciones domésticas y silvestres.

- Incluir dentro de la notificación obligatoria a especies y serovares de Salmonella como $S$. Typhimurium en casos de intoxicaciones alimentarias en humanos, ya que la presencia de esta bacteria en especies porcinas domésticas y silvestres es un factor relevante para la transmisión zoonótica.

- Reconocer el papel de la tuberculosis porcina en el mantenimiento de la infección en especies bovinas en el territorio nacional debería ser una prioridad para los entes de control que se encargan de la prevención y erradicación de esta enfermedad en especies de importancia zootécnica en Colombia.

\section{Literatura Citada}

1. Acevedo P, Jiménez-Valverde A, Aragón P, Niamir A. 2016. New developments in the study of species distribution. In: Current Trends in Wildlife Research. Springer. p 151-175.

2. Barandiaran S, Vivot MM, Pérez AM, Cataldi AA, Zumárraga MJ. 2015. Bovine tuberculosis in domestic pigs: Genotyping and distribution of isolates in Argentina. Res Vet Sci 103: 44-50. doi: 10.1016/j.rvsc.2015.09.013

3. Barasona JA, Latham MC, Acevedo P, Armenteros JA, Latham ADM, Gortazar C, Carro F, et al. 2014. Spatiotemporal interactions between wild boar and cattle: implications for cross-species disease transmission. Vet Res 45: 122. 
4. Borie CF, Jara MA, Sánchez ML, Martin BS, Arellano C, Martínez J, Prado V. 1997. Isolation and characterization of Yersinia enterocolitica from swine and cattle in Chile. Zoonoses Public Hlth 44: 347-354.

5. Cano-Terriza D, Risalde MÁ, Gortázar C, Infantes-Lorenzo JA, Domínguez, L, Domínguez M, et al. 2016. Estudio comparativo de la prevalencia de tubercolosis bovina en suidos domésticos y silvestres en el sur de España. [Internet]. Disponible en: https:// digital.csic.es/bitstream/10261/175682/1/ prevalenciaptuberculosuidos.pdf

6. Cárdenas JA. 2000. Situación en Colombia y Latinoamérica de las zoonosis. Revista MVZ Córdoba 5: 41-45. doi: 10.21897/rmvz.540

7. Carpinetti B, Castresana G Rojas P, Grant $J$, Marcos A, Monterubbianesi $M$ et al. 2014. Vigilancia epidemiológica en poblaciones de cerdos silvestres (Sus scrofa). Implicancias para salud pública, la producción animal y la conservación de la biodiversidad. SENASA. SNS 5-6: 67-76.

8. Carreras NB. 2020. Interacción patógeno-hospedador en la salmonelosis porcina. Modulación de la respuesta inmune intestinal por salmonella y mecanismos de persistencia Tesis Doctoral. Colombia: Univ de Córdoba. 238 p.

9. Carriquiriborde M, Milocco $\mathrm{SN}$, Príncipi G, Cagliada P, Carbone $C$. 2006. Pasteurella pneumotropica causa la regresión de tumores humanos trasplantados en ratones inmunodeficientes. Medicina 66: 242-244.

10. Center for Food Security and Public Health. 2009. Brucelosis porcina y rangiferina Brucella suis. [Internet]. Disponible en: http://www.cfsph.iastate.edu/ Factsheets/es/brucella_suis-es.pdf

11. Céspedes M. 2005. Leptospirosis: enfermedad zoonótica emergente. Rev Peru Med Exp Salud Pública 22: 290-307.

12. Chaparro PE, García I, Guerrero MI, León CI. 2004. Situación de la tuberculosis en Colombia, 2002. Biomédica 24: 102-114.
13. Chavarrias M. 2008. Detección de Yersinia enterocolítica. Fundación Eroski. [Internet]. Disponible en: http:// www.consumer.es/seguridad-alimentaria/ciencia-y-tecnologia/2008/11/19/ 181516.php

14. Chávez-de la Peña M, Higuera-Iglesias AL, Huertas-Jiménez MA, BáezMartínez R, Morales-de León J, Arteaga-Cabello F, Ponce de LeónRosales S. 2001. Brote por Salmonella enteritidis en trabajadores de un hospital. Salud Pública México 43: 211-216.

15. Cito F, Rijks J, Rantsios AT, Cunningham AA, Baneth G, Guardabassi L, Kuiken T. 2015. Prioritization of companion animal transmissible diseases for policy intervention in Europe. J Comp Pathol 155: S18-S26. doi: 10.1016/ j.jcpa.2015.01.007

16. Clark SO, Delogu G, Rayner E, Sali M, Williams A, Manganelli R. 2015. Improved protection in guinea pigs after vaccination with a recombinant $\mathrm{BCG}$ expressing MPT64 on its surface. Trials Vaccinol 4: 29-32. doi: 10.1016/ j.trivac.2015.03.003

17. De Busser EV, De Zutter L, Dewulf J, Houf K, Maes D. 2013. Salmonella control in live pigs and at slaughter. Vet J 196: 20-27. doi: 10.1016/j.tvj1.2013.01.002

18. De la Sota M, Belgrano D. 2004. Manual para veterinarios privados acreditados por SENASA. Enfermedades de los porcinos. Peste Porcina Clásica. Argentina: SENASA. $42 \mathrm{p}$.

19. [DNP] Departamento Nacional de Planeación. 2007. Documento Conpes. Política nacional de sanidad e inocuidad para la cadena porcícola. [Internet]. Disponible en: https://www.minambiente.gov.co/images/normativa/conpes/2007/ Conpes 3458 2007.pdf

20. Dolso MEI, Privado DPAUA. 2008. Avances en el control de enfermedades porcinas. En: IX Congreso Nacional de Producción Porcina. Argentina. 
21. Elika. 2013. Yersinia enterocolitica. Fundación Vasca para la Seguridad Agroalimentaria. [Internet]. Disponible en: https://www.elika.eus/bilatu/?selika=Yersinia

22. Espinal Marin P, Prieto Suárez E, Otero Jiménez, V, Máttar Velilla S. 2006. Presencia del gen de invasividad inv A en cepas de Salmonella spp: aisladas de alimentos del Caribe colombiano. Rev Cub Salud Pública 32. 116-120.

23. [FAO] Organización de las Naciones Unidas para la Alimentación y la Agricultura. 2010. Principales enfermedades de los cerdos. Programa especial de seguridad alimentaria. Nicaragua. 51 p. [Internet]. Disponible en: http:// www.fao.org/3/a-as540s.pdf

24. [FAO] Organización de las Naciones Unidas para la Agricultura y la Alimentación. 2012a. EMPRES - Boletín de enfermedades transfronterizas de los animales No. 41.

25. [FAO] Organización de las Naciones Unidas para la Alimentación y la Agricultura. 2012b. EMPRES Transboundary Animal Diseases. Bulletin No. 40. [Internet]. Disponible en: http:// www.fao.org/3/i2811e/i2811e.pdf

26. Ferreira AC, Almendra C, Cardoso R, Pereira MS, Beja-Pereira A, Luikart G, Corrêa de Sá MI. 2012. Development and evaluation of a selective medium for Brucella suis. Res Vet Sci 93: 565-567. doi: 10.1016/j.rvsc.2011.09.004

27. Ferro BE, Rodríguez AL, Pérez $M$, Travi BL. 2006. Seroprevalencia de infección por Leptospira en habitantes de barrios periféricos de Cali. Biomédica, 26: 250-257. doi: 10.7705/biomedica.v26i2.1414

28. Fredriksson-Ahomaa M, Wacheck $S$, Koenig M, Stolle A, Stephan R. 2009. Prevalence of pathogenic Yersinia enterocolitica and Yersinia pseudotuberculosis in wild boars in Switzerland. Int J Food Microb 135: 199-202. doi: 10.1016/j.ijfoodmicro.2009.08.019
29. Galindo RC, Muñoz PM, de Miguel MJ, Marin CM, Labairu J, Revilla M, Blasco JM, et al. 2010. Gene expression changes in spleens of the wildlife reservoir species, Eurasian wild boar (Sus scrofa), naturally infected with Brucella suis biovar 2. J Genet Genomics 37: 725-736. doi: 10.1016/S16738527(09)60090-4

30. Garzón MC, Angée DY, Llerena C, Orjuela DL, Victoria JE. 2008. Vigilancia de la resistencia del Mycobacterium tuberculosis a los fármacos antituberculosos, Colombia 2004-2005. Biomédica, 28: 319-326.

31. Hernández L. 2015. Problemas relativos a la calidad e inocuidad de los alimentos y su repercusión en el comercio. FAO. [Internet]. Disponible en: http:// www.fao.org/docrep/x $4390 \mathrm{t} /$ x4390t06.htm

32. Humblet MF, Vandeputte S, Albert A, Gosset C, Kirschvink N, Haubruge E, Fecher-Bourgeois H, et al. 2012. Multidisciplinary and evidence-based method for prioritizing diseases of food-producing animals and zoonoses. Emerg Infect Dis 18: e1. doi: 10.3201/eid1804.-111151

33. [ICA] Instituto Colombiano Agropecuario. 2011. Colombia: Sanidad Animal 2011. Situación epidemiológica en Colombia en cuanto a las enfermedades de la lista de la Organización Mundial de Sanidad Animal (OIE). [Internet]. Disponible en: https://www.ica.gov.co/ getattachment/b66f6f33-43bb-4c2ca8f6-e66ab31194e0/2011.aspx

34. [ICA] Instituto Colombiano Agropecuario. 2021. Censo pecuario nacional. 2021. [Internet]. Disponible en: https:// www.ica.gov.co/areas/pecuaria/servicios/epidemiologia-veterinaria/censos2016/censo-2018

35. Iglesias E. 2014. Evaluación del riesgo asociado a la presencia de bacterias patógenas en carnes frescas y productos cárnicos listos para el consumo comercializados en la ciudad de León. Tesis Doctoral. León, España: Univ. de León. $234 \mathrm{p}$. 
36. [INS] Instituto Nacional de Salud. 2015. Boletín epidemiológico semanal. Semana epidemiológica $\mathrm{N}^{\mathrm{o}} 52$ de 2015. [Internet]. Disponible en: https:// www.ins.gov.co/buscador-eventos/ BoletinEpidemiologico/2015\%20Boletin\%20epidemiologico\%20Semana\%2052.pdf

37. [INS] Instituto Nacional de Salud. 2016. Boletín epidemiológico semanal. Semana epidemiológica $\mathbf{N}^{0} 28 \mathrm{de}$ 2016. [Internet]. Disponible en: https:// www.ins.gov.co/buscador-eventos/ BoletinEpidemiologico/2016\%-20Bolet\%C3\%ADn\%20epidemiol\%C3\%B3gico\%20semana\%-2028.pdf

38. [INS] Instituto Nacional de Salud. 2017. Guía para la vigilancia por laboratorio del Leptospira spp. Dirección redes en salud pública subdirección laboratorio nacional de referencia grupo de microbiología 2017. [Internet]. Disponible en: https://www.ins.gov.co/buscador/Informacin\%20de\%20laboratorio/ Gu\%C3\%ADa\%20para\%201a\%20vigilancia \%2-0por\%201aboratorio\%20de\%20Leptospira\%20spp.pdf

39. [INS] Instituto Nacional de Salud. 2019a. Informe del Evento. Enfermedades Transmitidas Por Alimentos Colombia 2019. [Internet]. Disponible en: https://www.ins.gov.co/buscador-eventos/Informesdeevento/ENFERMEDADES\%20TRANSMITIDAS\%-20POR\%2-0ALIMENTOS_2019.pdf

40. INS] Instituto Nacional de Salud. 2019b. Boletín Epidemiológico Semanal. Semana epidemiológica 52. [Internet]. Disponible en: https://www.ins.gov.co/ buscador-eventos/Boletin Epidemiologico/2019_Boletin_-epidemiologico_semana_52.pdf

41. Jaramillo L, Arboleda M, García V, Agudelo-Flórez P. 2014. Coinfección brucelosis-leptospirosis, Urabá, Colombia. Reporte de caso. Infectio 18: 72-76.

42. Jofré M, Perret P, Abarca V, Solari G, Olivares C, López J. 2006. Recomendaciones para el manejo de mordeduras ocasionadas por animales. Rev Chilena Infectol 23: 20-34.
43. Kopper G, Calderón G, Schneider S, Domínguez W, Gutiérrez G, Rosell C, Mejía D. 2009. Enfermedades transmitidas por alimentos y su impacto socioeconómico. Estudios de caso en Costa Rica, El Salvador, Guatemala, Honduras y Nicaragua. FAO. [Internet]. Disponible en: http://www.fao.org/publications/ card/en/c/75dfb832-0336-5cd3-8433$9 \mathrm{~cd} 28115 \mathrm{e} 18 \mathrm{f} /$

44. Krause G, Working Group on Prioritization at Robert Koch Institute. 2008. How can infectious diseases be prioritized in public health? a standardized prioritization scheme for discussion. EMBO Rep 9(Suppl 1): 22-27. doi: 10.1038/embor.2008.76

45. Kumar H, Mahajan V, Sharma S, Singh R, Arora AK, Banga HS, Verma $S$, et al. 2007. Concurrent pasteurellosis and classical swine fever in Indian pigs. J Swine Health Prod 15: 279-283.

46. León A. 1997. Contribución a la epidemiologia molecular de Pasteurella multocida tipo A mediante el estudio de la huella genómica. Tesis Doctoral. Bogotá, Colombia: Pontificia Universidad Javeriana. $100 \mathrm{p}$.

47. Leotta GA, Vigo GB, Chinen I, Prieto M, Callejo R, Rivas M. 2006. Identificación, biotipificación y caracterización de cepas de Pasteurella multocida aisladas en la Argentina. Rev Argentina Microbiol 38: 125-129.

48. Luna MA, Morales LP, Torres JI, Gual F. 1996. Investigación serológica de leptospirosis en fauna silvestre mantenida en cautiverio en el zoológico de Chapultepec de la ciudad de México. Vet Mexico 3: 229-234.

49. Marín ML. 2020. Zoonosis y determinantes sociales de la salud: scoping review. Tesis de Administradora en Salud. Colombia: Univ. de Antioquia. 109 p.

50. Martínez R, Pérez A, Baró M, Álvarez AM, Menéndez J, Díaz M, Cruz R, et al. 2000. Evaluación de la efectividad de una nueva vacuna contra la leptospirosis humana en grupos de riesgo. Rev Panam Salud Pública 8: 385-392. 
51. Martín-Hernando MP, Höfle U, Vicente J, Ruiz-Fons F, Vidal D, Barral M, Garrido JM, et al. 2007. Lesions associated with Mycobacterium tuberculosis complex infection in the European wild boar. Tuberculosis 87: 360-367. doi: 10.1016/j.tube.2007.02.003

52. Máttar S, Visbal J, Bermúdez A. 2000. Zoonosis: ¿cerca o lejos de nosotros? Revista MVZ Córdoba 5: 5-9. doi: 10.21897/rmvz.548

53. Millán Pérez LF. 2015. Evaluación serológica frente a Salmonella spp, Yersinia spp y Leptospira spp, con potencial zoonótico en granjas porcícolas de Cundinamarca. Colombia. Tesis de Grado. Bogotá, Colombia: Pontifica Universidad Javeriana. $40 \mathrm{p}$.

54. Miller RS, Farnsworth ML, Malmberg JL. 2013. Diseases at the livestockwildlife interface: status, challenges, and opportunities in the United States. Prev Vet Med 110: 119-132. doi: 10.1016/ j.prevetmed.2012.11.021

55. Ministerio de Salud. 2014. Enfermedades infecciosas. Leptospirosis. Diagnóstico de Leptospirosis. Guía para el equipo de salud. República Argentina. [Internet]. Disponible en: https:// www.entrerios.gov.ar/msalud/wpcontent/uploads/2018/05/guia-medicaleptospirosis.pdf

56. Moles-Cervantes LP, UrrutiaVelázquez RM, Diosdado-Vargas F, Morilla-González A. 1998. Frecuencia de Leptospira interrogans en unidades de producción porcina del altiplano de México. Vet México 29: 49.

57. Nájera S, Alvis N, Babilonia D, Alvarez L, Máttar S. 2005. Leptospirosis ocupacional en una región del Caribe colombiano. Salud Pública México 47: 240-244.

58. Ng V, Sargeant JM. 2013. A quantitative approach to the prioritization of zoonotic diseases in North America: a health professionals' perspective. PLoS One 8: e72172. doi: 10.1371/journal.pone. 0072172
59. Noreña EA, Galvis KL. 2020. Oportunidades de mejora para la actualización de la guía ambiental subsector porcícola en Colombia - caso de estudio en el departamento de Risaralda. Tesis de Ingeniero Ambiental. Colombia: Universidad Nacional Abierta y a Distancia - UNAD.121 p.

60. Ochoa JE, Sánchez A, Ruiz I. 2000. Epidemiología de la leptospirosis en una zona andina de producción pecuaria. Rev Panam Salud Pública 7. 325-331.

61. [OIE] The World Organization for Animal Health. 2016. 84SG Sesión General. Informe final 2016. [Internet]. Disponible en: https://www.oie.int/app/ uploads/2021/03/e-if-2016-public.pdf

62. [OMS] Organización Mundial de la Salud. 2008. Leptospirosis humana: guía para el diagnóstico, vigilancia y control. Serie de Manuales Técnicos 12. Rio de Janeiro, Brasil.

63. Otte J, Pica-Ciamarra U. 2021. Emerging infectious zoonotic diseases: the neglected role of food animals. One Health: 100323. doi: 10.1016/j.onehlt.2021.100323

64. Parra A, Fernández-Llario P, Tato A, Larrasa J, García A, Alonso JM, Hermoso de Mendoza M, Hermoso de Mendoza J. 2003. Epidemiology of Mycobacterium bovis infections of pigs and wild boars using a molecular approach. Vet Microbiol 97: 123-133. doi: 10.1016/j.vetmic.2003.08.007

65. Pulido-Villamarín A, CastañedaSalazar R, Mendoza-Gómez MF, Vivas-Díaz L. 2019. Presencia de anticuerpos frente a algunos patógenos de interés zoonótico en cuatro granjas porcícolas de Cundinamarca, Colombia. Rev Inv Vet Perú 30: 446-454. doi: 10.15381/rivep.v30i1.15702

66. Ramírez E. 2014. Situación actual de la leptospirosis porcina en México. Tesis de Médico Veterinario Zootecnista. Torreón, México: Univ. Autónoma Agraria Antonio Narro. $16 \mathrm{p}$. 
67. Reyes BAS, Tagle JAMG, Mendía RJ. 2009. Pneumonia by Pasteurella multocida in an adolescent patient. A case report. Enferm Infec Micr 29: 81-85.

68. Risco D, Fernández-Llario P, Cuesta JM, García-Jiménez WL, Gil M, Gonçalves P, Martínez R, et al. 2013. Fatal outbreak of systemic pasteurellosis in a wild boar (Sus scrofa) population from southwest Spain. J Vet Diagn Invest 25: 791-794. doi: 10.1177/10406387135-04411

69. Rist CL, Arriola CS, Rubin C. 2014. Prioritizing zoonoses: a proposed one health tool for collaborative decisionmaking. PLoS One 9: e109986. doi: 10.1371/journal.pone.0109986

70. Rosner BM, Stark K, Werber D. 2010. Epidemiology of reported Yersinia enterocolitica infections in Germany, 2001-2008. BMC Public Health 10: 337. doi: 10.1186/1471-2458-10-337

71. Rovid A, Roth AJ, Galyon J, Lofstedt J, Lenardón MV. 2010. Enfermedades emergentes y exóticas de los animales. USA: Iowa State University. 336 p.

72. Sánchez M. 1990. Control sanitario y planificación preventiva en explotaciones porcinas. Mundo ganadero 8: 42-45.

73. Sánchez-Jiménez MM, GiraldoEcheverri CA, Olivera-Angel $M$. 2013. Infección por Brucella canis en humanos: propuesta de un modelo teórico de infección a través de la ruta oral. Infectio 17: 193-200. doi: 10.1016/S01239392(13)70731-8

74. Sánchez-Jiménez MM, Isaza JP, Alzate JF, Olivera-Angel M. 2015. Comparison of Brucella canis genomes isolated from different countries shows multiple variable regions. Genomics 106: 43-51. doi: 10.1016/j.ygeno.2015.03.004

75. [SENASA] Servicio Nacional de Sanidad y Calidad Agroalimentaria. 2009. Manual para veterinarios privados acreditados por Senasa. Enfermedades de los porcinos. Argentina: SENASA. $46 \mathrm{p}$.
76. Sepúlveda A, Santiago J, Preciado FJ. 2002. La rata y el perro, importantes vectores de la leptospirosis en explotaciones pecuarias de Cd. Guzmán, Jalisco. Rev Cubana Med Trop 54: 21-23.

77. Shayegani M, Stone WB, DeForge I, Root T, Parsons LM, Maupin P. 1986. Yersinia enterocolitica and related species isolated from wildlife in New York State. Appl Environ Microbiol 52: 420-442. doi: 10.1128/AEM.52.3.420424.1986

78. Signorini M, Civit S, Bonilla $M$, Cervantes M, Calderón M, Pérez A, et al. 2006. Evaluación de riesgos de los rastros y mataderos municipales. México: Comisión Federal para la Protección de Riesgos Sanitarios. 32 p.

79. Smego R, Gallis H. 1984. Pasteurelosis. Rev infect Dis 6: 164-180.

80. Stoffregen WC, Johnson CS, Olsen $S C$. 2013. Immunogenicity and safety of a natural rough mutant of Brucella suis as a vaccine for swine. Res Vet Sci 95: 451-458. doi: 10.1016/j.rvsc.2013.-04.014

81. Varón M. 2020. Importancia de Salmonellla en porcinos y su diagnóstico. Tesis de Grado. Colombia: Univ. Cooperativa de Colombia.

82. [WAHIS] Wild Animal Health Information. s.f. Sistema mundial de seguimiento de las enfermedades de los animales salvajes de la OIE. [Internet]. Disponible en: https://wahis.oie.int/\#/home

83. Zottola T, Montagnaro S, Magnapera C, Sasso S, De Martino L, Bragagnolo A, D'Amici L, et al. 2013. Prevalence and antimicrobial susceptibility of salmonella in European wild boar (Sus scrofa); Latium Region - Italy. Comp Immunol Microb 36: 161-168. doi: 10.1016/j.cimid.2012.11.004 